\title{
Some Upper Matrix Bounds for the Solution of the Continuous Algebraic Riccati Matrix Equation
}

\author{
Zübeyde Ulukök and Ramazan Türkmen \\ Department of Mathematics, Science Faculty, Selçuk University, 42031 Konya, Turkey \\ Correspondence should be addressed to Zübeyde Ulukök; zulukok@selcuk.edu.tr
}

Received 22 May 2013; Accepted 24 October 2013

Academic Editor: Baolin Wang

Copyright ( 2013 Z. Ulukök and R. Türkmen. This is an open access article distributed under the Creative Commons Attribution License, which permits unrestricted use, distribution, and reproduction in any medium, provided the original work is properly cited.

\begin{abstract}
We propose diverse upper bounds for the solution matrix of the continuous algebraic Riccati matrix equation (CARE) by building the equivalent form of the CARE and using some matrix inequalities and linear algebraic techniques. Finally, numerical example is given to demonstrate the effectiveness of the obtained results in this work as compared with some existing results in the literature. These new bounds are less restrictive and provide more efficient results in some cases.
\end{abstract}

\section{Introduction}

In many areas of optimal control [1-3], robust control [1], robust stability [4], filter design [5], stability theory and analysis [6-8], control design [9] in control theory $[10,11]$ including optimization stability theory, and transient performance performance nonlinear systems [12], the algebraic Riccati and Lyapunov matrix equations play an important role.

For example, consider the following linear system such that $A \in \mathbb{R}^{n \times n}, B \in \mathbb{R}^{n \times m}, x_{0} \in \mathbb{R}^{n}[13]$ :

$$
\begin{gathered}
\dot{x}(t)=A x(t)+B u(t), \\
x(0)=x_{0},
\end{gathered}
$$

with the state feedback control

$$
u(t)=-K x(t), \quad K=B^{T} P,
$$

and the performance index

$$
J=\int_{0}^{\infty}\left(x^{T} Q x+u^{T} u\right) d t
$$

where $R=B B^{T} \in \mathbb{R}^{n \times n}, Q \in \mathbb{R}^{n \times n}$ is positive semidefinite matrix, and $P$ is the positive semidefinite solution to the continuous algebraic Riccati matrix equation (CARE)

$$
A P+P A^{T}-P R P+Q=0 .
$$

When $B=0$ and $A$ is stable matrix, the CARE (4) becomes the continuous algebraic Lyapunov matrix equation (CALE)

$$
A^{T} P+P A=-Q .
$$

It is assumed that the pair $\left(A, R^{1 / 2}\right)$ is stabilizable. Then the CARE (4) has a unique symmetric positive semidefinite stabilizing solution if the pair $\left(A, Q^{1 / 2}\right)$ is observable.

The problem of estimating solution bounds for the algebraic Riccati and Lyapunov matrix equations has widely been considered in the recent years, since these equations are widely used in many fields of control system analysis and design. A number of works have reported numerical algorithms to get the exact solution of the mentioned equations [7]. However, we should note that the analytical solution of these equations has some complications and computational burdens, specially, when the dimensions of the system matrices increase. Thus, for some applications such as stability analysis [8], it is the only preferred solution matrix bounds for the exact solution that can be obtained without hard and complicated burdens. Moreover, as mentioned in [12], in practice, the solution matrix bounds can also be used as approximations of the exact solution or initial guesses in the numerical algorithms for the exact solution [10].

The existing results obtained during 1974-1994 have been summarized by Kwon et al. [14] only including all eigenvalue bounds such as the extreme eigenvalues, the summation, 
the trace, majorization inequalities, the product, and the determinant. Unfortunately, by this time, the upper matrix bounds for the solution of the CARE (4) have not been proposed in the literature. However, Lee in [15] has proposed upper and lower matrix bounds for the CARE (4) and henceforth many reports have been presented for the upper [16-20] and lower $[18,19,21]$ bounds for the solution of the CARE (4). As matrix bounds include all eigenvalue bounds [14, 22, 23] particularly the minimum and maximum eigenvalues, trace $[10,24,25]$, determinant [14], and norm [26] bounds, it is seen that they are the most general and useful. Therefore, this paper presents upper matrix bounds for the solution of the CARE (4) by utilizing various matrix identities and matrix inequalities.

Let $\mathbb{R}^{n \times m}$ be the set of $n \times m$ real matrices. In this paper, we denote the eigenvalues of an $n \times n$ real matrix by $\lambda_{i}(X)$; if $X \in$ $\mathbb{R}^{n \times n}$ is a symmetric matrix, then its eigenvalues are arranged in the nonincreasing order $\lambda_{1}(X) \geq \lambda_{2}(X) \geq \cdots \geq \lambda_{n}(X)$. For $X \in \mathbb{R}^{n \times m}$, suppose that the singular values of $X$ are ordered in nonincreasing form; that is, $s_{1}(X) \geq s_{2}(X) \geq \cdots \geq$ $s_{n}(X)$. Also, let $\operatorname{tr}(X), X^{T}, X^{-1}$, and $\operatorname{det}(X)$ denote the trace, transpose, inverse, determinant, respectively. Additionally, the spectral condition number of any matrix $X$ is defined by $\kappa_{2}(X)=s_{1}(X) s_{1}\left(X^{-1}\right)$. Write $X \geq(>) 0$, if $X$ is a positive semidefinite (positive definite) matrix. For the symmetric matrices of the same size $X$ and $Y$, if $X-Y$ is positive semidefinite, we write $X \geq Y$ or $Y \leq X$. Then, Weyl's monotonicity principle means that $Y \leq X$ leads to $\lambda_{i}(Y) \leq \lambda_{i}(X)$, $i=1,2, \ldots, n$. The identity matrix in $\mathbb{R}^{n \times n}$ is shown by $I$.

The following lemmas are used to prove the main result of this paper.

Lemma 1 (see $[27,28]$ ). Let $X \in \mathbb{R}^{n \times n}$ be symmetric matrix. Then the following inequality holds:

$$
\lambda_{n}(X) I \leq X \leq \lambda_{1}(X) I
$$

Lemma 2 (see $[27,28]$ ). For any matrix $A \in \mathbb{R}^{n \times m}$ and any positive semidefinite matrices $X, Y \in \mathbb{R}^{n \times n}$ such that $X \geq Y>$ $(\geq) 0$, it holds that $A^{T} X A \geq A^{T} Y A$, with strict inequality if $X$ and $Y$ are positive definite and $A$ is of full rank.

Lemma 3 (see $[27,28]$ ). For any symmetric matrices $X, Y \in$ $\mathbb{R}^{n \times n}$, the following inequality holds:

$$
\begin{array}{r}
\lambda_{i+j-1}(X+Y) \leq \lambda_{j}(X)+\lambda_{i}(Y), \\
i+j \leq n+1, \quad 1 \leq i, \quad j \leq n .
\end{array}
$$

Lemma 4 (see [28]). Let $X, Y \in \mathbb{R}^{n \times n}$, for $i=1,2, \ldots, n$, one has

$$
s_{i}(X+Y) \leq s_{i}(X)+s_{1}(Y)
$$

Lemma 5 (see [28]). Let $X \in \mathbb{R}^{m \times n}, Y \in \mathbb{R}^{n \times m}$, for $i=$ $1,2, \ldots, n$, one has

$$
s_{i}(X Y) \leq s_{i}(X) s_{1}(Y) .
$$

Lemma 6 (see [29]). Let $X \in \mathbb{R}^{n \times n}$, for $i=1,2, \ldots, n$, then

$$
\left|\lambda_{i}\left(\frac{A+A^{T}}{2}\right)\right| \leq s_{i}(A) .
$$

Lemma 7 (see [30]). The following matrix inequality:

$$
\left(\begin{array}{cc}
W & S \\
S^{T} & V
\end{array}\right)>0
$$

where $W=W^{T}$ and $V=V^{T}$, is equivalent to either

$$
V>0, \quad W-S V^{-1} S^{T}>0,
$$

or

$$
W>0, \quad V-S^{T} W^{-1} S>0 .
$$

Lemma 8 (see [17]). The positive semidefinite solution $P$ of the CARE (4) has the following upper bound on its maximal eigenvalue:

$$
\lambda_{1}(P) \leq \lambda_{1}\left(D^{T} D\right) \frac{\lambda_{1}\left[\left(Q+K^{T} K\right) D^{T} D\right]}{\lambda_{n}\left(Z D^{T} D\right)} \equiv \eta
$$

where $K$ is any matrix stabilizing $A+B K$ (i.e., $\operatorname{Re}\left(\lambda_{i}(A+B K)\right)<$ 0 for all $i)$ and the nonsingular matrix $D$ and positive definite matrix $Z$ are chosen to yield the LMI

$$
(A+B K)^{T} D^{T} D+D^{T} D(A+B K) \leq-Z .
$$

This eigenvalue upper bound (14) is always calculated if there exists a unique positive semidefinite solution of the CARE (4).

\section{Main Results}

Zhang and Liu in [19] obtained the lower and upper bounds for the solution of the CARE (4) which improve the results in [21]. Also, Lee in [18] proposed upper and lower bounds for the solution of the CARE (4) by considering a different approach. In this section, we will present diverse upper matrix bounds for the solution matrix of the CARE (4) in the light of the reported results in $[18,19]$, by utilizing the above lemmas and generating some matrix identities.

Theorem 9. Assume that $Q$ is symmetric positive definite and there exists a unique symmetric positive semidefinite solution $P$ to the CARE (4). Then P satisfies the following inequality:

$$
\begin{aligned}
P \leq & \left\{\frac{1}{1-\lambda_{1}(I-\varepsilon R)}\right. \\
& \left.\times\left\{M+2 \varphi_{1} \varepsilon \lambda_{1}(I-\varepsilon R) s_{1}\left[A(I-\varepsilon R)^{-1}\right] I\right\}\right\}^{1 / 2} \\
\equiv & P_{u 1}(\varepsilon, A, Q, R)
\end{aligned}
$$


where the positive semidefinite matrix $M_{1}$ and the positive constant $\delta$ are defined by

$$
\begin{aligned}
M= & \varepsilon Q-\varepsilon^{2} A(I-\varepsilon R)^{-1} A^{T} \\
& +\varepsilon^{2} \lambda_{1}(I-\varepsilon R) A(I-\varepsilon R)^{-2} A^{T}, \\
\delta= & \frac{\varepsilon \lambda_{1}(I-\varepsilon R) s_{1}\left[A(I-\varepsilon R)^{-1}\right]}{1-\lambda_{1}(I-\varepsilon R)},
\end{aligned}
$$

where $\varepsilon$ is any positive constant such that

$$
0<\varepsilon<\left\|R+A^{T} Q^{-1} A\right\|^{-1},
$$

and positive constant $\varphi_{1}$ is defined by

$$
\begin{aligned}
\varphi_{1} \equiv & \frac{1}{1-\lambda_{1}(I-\varepsilon R)} \\
& \times\left\{\left\{\left[1-\lambda_{1}(I-\varepsilon R)\right] \lambda_{1}(M)+\delta^{2}\right\}^{1 / 2}+\delta\right\} .
\end{aligned}
$$

Proof. By adding and subtracting $(1 / \varepsilon) P P+A((1 / \varepsilon)(I-$ $R))^{-1} A^{T}$ from (4), one gets

$$
\begin{aligned}
& {\left[P+A\left(\frac{1}{\varepsilon} I-R\right)^{-1}\right]\left(\frac{1}{\varepsilon} I-R\right)\left[P+A\left(\frac{1}{\varepsilon} I-R\right)^{-1}\right]^{T}} \\
& -\frac{1}{\varepsilon} P P-A\left(\frac{1}{\varepsilon} I-R\right)^{-1} A^{T}+Q=0,
\end{aligned}
$$

therefore,

$$
\begin{aligned}
& {\left[P+A\left(\frac{1}{\varepsilon} I-R\right)^{-1}\right]\left(\frac{1}{\varepsilon} I-R\right)\left[P+A\left(\frac{1}{\varepsilon} I-R\right)^{-1}\right]^{T}} \\
& \quad=\frac{1}{\varepsilon} P P+A\left(\frac{1}{\varepsilon} I-R\right)^{-1} A^{T}-Q .
\end{aligned}
$$

Applying Lemmas 1 and 2 to (21) gives

$$
\begin{aligned}
\frac{1}{\varepsilon} P P & +A\left(\frac{1}{\varepsilon} I-R\right)^{-1} A^{T}-Q \\
= & {\left[P+A\left(\frac{1}{\varepsilon} I-R\right)^{-1}\right]\left(\frac{1}{\varepsilon} I-R\right) } \\
& \times\left[P+A\left(\frac{1}{\varepsilon} I-R\right)^{-1}\right]^{T} \\
\leq & \lambda_{1}\left(\frac{1}{\varepsilon} I-R\right)\left[P+A\left(\frac{1}{\varepsilon} I-R\right)^{-1}\right] \\
& \times\left[P+A\left(\frac{1}{\varepsilon} I-R\right)^{-1}\right]^{T} \\
\leq & \lambda_{1}\left(\frac{1}{\varepsilon} I-R\right)\left[P^{2}+A\left(\frac{1}{\varepsilon} I-R\right)^{-2} A^{T}\right. \\
& \left.+P\left(\frac{1}{\varepsilon} I-R\right)^{-1} A^{T}+A\left(\frac{1}{\varepsilon} I-R\right)^{-1} P\right] .
\end{aligned}
$$

For the part $P((1 / \varepsilon) I-R)^{-1} A^{T}+A((1 / \varepsilon) I-R)^{-1} P$ of (22), applying Lemmas 1,6 , and 5 , respectively, shows that

$$
\begin{aligned}
& P\left(\frac{1}{\varepsilon} I-R\right)^{-1} A^{T}+A\left(\frac{1}{\varepsilon} I-R\right)^{-1} P \\
& \quad \leq \lambda_{1}\left(P\left(\frac{1}{\varepsilon} I-R\right)^{-1} A^{T}+A\left(\frac{1}{\varepsilon} I-R\right)^{-1} P\right) I \\
& \quad \leq\left|\lambda_{1}\left(P\left(\frac{1}{\varepsilon} I-R\right)^{-1} A^{T}+A\left(\frac{1}{\varepsilon} I-R\right)^{-1} P\right) I\right| \\
& \quad \leq 2 s_{1}\left(P\left(\frac{1}{\varepsilon} I-R\right)^{-1} A^{T}\right) I \\
& \quad \leq 2 \lambda_{1}(P) s_{1}\left(A\left(\frac{1}{\varepsilon} I-R\right)^{-1}\right) I .
\end{aligned}
$$

Thus, in light of the fact (23), (22) becomes

$$
\begin{aligned}
\frac{1}{\varepsilon} P P & +A\left(\frac{1}{\varepsilon} I-R\right)^{-1} A^{T}-Q \\
\leq \lambda_{1}\left(\frac{1}{\varepsilon} I-R\right)[ & P^{2}+A\left(\frac{1}{\varepsilon} I-R\right)^{-2} A^{T} \\
& \left.+2 \lambda_{1}(P) s_{1}\left(A\left(\frac{1}{\varepsilon} I-R\right)^{-1}\right) I\right] .
\end{aligned}
$$

If $Q>0$ and $\varepsilon$ satisfies (18), then

$$
I-\varepsilon R-\varepsilon A^{T} Q^{-1} A>0, \quad \varepsilon Q>0 .
$$

By the application of the Schur complement formula of Lemma 7 to (25), we can say that the above inequalities are satisfied if and only if

$$
\left(\begin{array}{cc}
\varepsilon Q & \varepsilon A \\
\varepsilon A^{T} & I-\varepsilon R
\end{array}\right)>0
$$

which means that

$$
\varepsilon Q-\varepsilon^{2} A(I-\varepsilon R) A^{T}>0, \quad I-\varepsilon R>0 .
$$

Therefore, we say that (24) is equivalent to

$$
\begin{aligned}
P^{2} \leq & \lambda_{1}(I-\varepsilon R)\left[P^{2}+A\left(\frac{1}{\varepsilon} I-R\right)^{-2} A^{T}\right. \\
& \left.+2 \lambda_{1}(P) s_{1}\left(A\left(\frac{1}{\varepsilon} I-R\right)^{-1}\right) I\right] \\
& +\varepsilon Q-\varepsilon^{2} A(I-\varepsilon R)^{-1} A^{T} .
\end{aligned}
$$

Since $1-\lambda_{1}(I-\varepsilon R)>0$, (28) can be rewritten as

$$
\begin{aligned}
& P^{2} \leq \frac{1}{1-} \lambda_{1}(I-\varepsilon R) \\
& \times\left\{\varepsilon Q-\varepsilon^{2} A(I-\varepsilon R)^{-1} A^{T}\right. \\
& \quad+\varepsilon^{2} \lambda_{1}(I-\varepsilon R) A(I-\varepsilon R)^{-2} A^{T} \\
&\left.\quad+2 \lambda_{1}(P) \varepsilon \lambda_{1}(I-\varepsilon R) s_{1}\left[A(I-\varepsilon R)^{-1}\right] I\right\} .
\end{aligned}
$$


Utilizing the relations in Lemmas 1 and 3, (29) becomes

$$
\begin{aligned}
& \lambda_{1}\left(P^{2}\right) \\
& \leq \frac{1}{1-\lambda_{1}(I-\varepsilon R)} \lambda_{1} \\
& \quad \times\left\{\left[\varepsilon Q-\varepsilon^{2} A(I-\varepsilon R)^{-1} A^{T}\right.\right. \\
& \left.\quad+\varepsilon^{2} \lambda_{1}(I-\varepsilon R) A(I-\varepsilon R)^{-2} A^{T}\right] \\
& \left.\quad+2 \lambda_{1}(P) \varepsilon \lambda_{1}(I-\varepsilon R) s_{1}\left[A(I-\varepsilon R)^{-1}\right]\right\} \\
& \leq \frac{1}{1-\lambda_{1}(I-\varepsilon R)} \\
& \quad \times\left\{\lambda _ { 1 } \left[\varepsilon Q-\varepsilon^{2} A(I-\varepsilon R)^{-1} A^{T}\right.\right. \\
& \left.\quad+\varepsilon^{2} \lambda_{1}(I-\varepsilon R) A(I-\varepsilon R)^{-2} A^{T}\right] \\
& \left.\quad+2 \lambda_{1}(P) \varepsilon \lambda_{1}(I-\varepsilon R) s_{1}\left[A(I-\varepsilon R)^{-1}\right]\right\} .
\end{aligned}
$$

Solving (30) according to $\lambda_{1}(P)$ gives

$$
\begin{aligned}
& \lambda_{1}(P) \leq \frac{1}{1-\lambda_{1}(I-\varepsilon R)} \lambda_{1} \\
& \times\left[\varepsilon Q-\varepsilon^{2} A(I-\varepsilon R)^{-1} A^{T}\right. \\
&\left.+\varepsilon^{2} \lambda_{1}(I-\varepsilon R) A(I-\varepsilon R)^{-2} A^{T}\right] \\
&\left.+\left[\frac{\varepsilon \lambda_{1}(I-\varepsilon R) s_{1}\left[A(I-\varepsilon R)^{-1}\right]}{1-\lambda_{1}(I-\varepsilon R)}\right]^{2}\right\}^{1 / 2} \\
&+\frac{\varepsilon \lambda_{1}(I-\varepsilon R) s_{1}\left[A(I-\varepsilon R)^{-1}\right]}{1-\lambda_{1}(I-\varepsilon R)} \equiv \varphi_{1} .
\end{aligned}
$$

Substituting (31) into (29) results in the upper bound

$$
\begin{aligned}
& P \leq\left\{\frac{1}{1-\lambda_{1}(I-\varepsilon R)}\right. \\
& \quad \times\left\{\varepsilon Q-\varepsilon^{2} A(I-\varepsilon R)^{-1} A^{T}\right. \\
& \quad+\varepsilon^{2} \lambda_{1}(I-\varepsilon R) A(I-\varepsilon R)^{-2} A^{T} \\
&\left.\left.\quad+2 \varphi_{1} \varepsilon \lambda_{1}(I-\varepsilon R) s_{1}\left[A(I-\varepsilon R)^{-1}\right] I\right\}\right\}^{1 / 2} .
\end{aligned}
$$

This completes the proof.

Remark 10. The inequality (3.5) in [19] is clearly as follows:

$$
\begin{aligned}
P^{2} \leq & \lambda_{1}(I-\varepsilon R)\left\{\lambda_{1}^{2}(P)+2 \lambda_{1}(P) \varepsilon s_{1}\left[A(I-\varepsilon R)^{-1}\right]\right. \\
& \left.+\varepsilon^{2} s_{1}^{2}\left[A(I-\varepsilon R)^{-1}\right]\right\} \\
& +\varepsilon Q-\varepsilon^{2} A(I-\varepsilon R)^{-1} A^{T} .
\end{aligned}
$$

Thus, when the inequality (28) is considered, from the facts

$$
\begin{gathered}
P^{2} \leq \lambda_{1}^{2}(P), \\
{\left[A(I-\varepsilon R)^{-1}\right]\left[A(I-\varepsilon R)^{-1}\right]^{T} \leq s_{1}^{2}\left[A(I-\varepsilon R)^{-1}\right],}
\end{gathered}
$$

it is seen that the upper bound in Theorem 9 is always sharper than the result given by Theorem 3.1 in [19].

Remark 11. It is well known that most of the studies in the literature have focused to derive the bounds for the maximum and minimum eigenvalues, the trace, and the determinant for the solution of the CARE (4); however, the matrix solution bounds are quite restriction. Among the mentioned bounds, the matrix solution bounds are the most useful and efficient because other bounds that are dependent on eigenvalue can be derived directly from matrix solution bounds via monotonicity.

By using Theorem 9, we can derive the following result immediately.

Corollary 12. Assume that $Q$ is symmetric positive definite and there exists a unique symmetric positive semidefinite solution $P$ to the CARE (4). Then P satisfies the following upper eigenvalue bounds:

$$
\begin{aligned}
\lambda_{i}(P) & \leq \min _{\varepsilon} \lambda_{i}\left(P_{u 1}(\varepsilon, A, Q, R)\right)=P_{u 1}^{1} \\
& \leq \lambda_{i}\left(P_{u 1}(\varepsilon, A, Q, R)\right) \\
\operatorname{tr}(P) & \leq \sum_{i=1}^{n} P_{u 1}^{1} \leq \min _{\varepsilon} \operatorname{tr}\left(P_{u 1}(\varepsilon, A, Q, R)\right) \\
& \leq \operatorname{tr}\left(P_{u 1}(\varepsilon, A, Q, R)\right), \\
\operatorname{det}(P) & \leq \prod_{i=1}^{n} P_{u 1}^{1} \leq \min _{\varepsilon} \operatorname{det}\left(P_{u 1}(\varepsilon, A, Q, R)\right) \\
& \leq \operatorname{det}\left(P_{u 1}(\varepsilon, A, Q, R)\right),
\end{aligned}
$$

where $\varepsilon$ satisfies (18) and $P_{u 1}$ is defined by (16).

By establishing the more general form than the matrix identity used in Theorem 9 for the CARE (4), one gets the following upper bounds.

Theorem 13. Let $X$ be any symmetric positive definite matrix. Then the unique symmetric positive semidefinite solution $P$ to the CARE (4) has the following upper bound

$$
\begin{aligned}
P \leq M_{1}^{-1 / 2}\left\{M _ { 1 } ^ { 1 / 2 } \left[Q-X_{1}+\lambda_{1}\left(X_{1}^{-1}\right)\right.\right. & \\
& \left.\times\left[\eta s_{1}(A)+\lambda_{1}\left(X_{1}\right)\right]^{2} I\right] \\
& \left.\times M_{1}^{1 / 2}\right\}^{1 / 2} M_{1}^{-1 / 2} \\
\equiv P_{u 2}\left(\eta, X_{1}, A, Q, R\right) &
\end{aligned}
$$

where the positive definite matrix $X_{1}$ is chosen so that

$$
M_{1} \equiv A^{T} X_{1}^{-1} A+R>0,
$$

and $\eta$ is defined by (14). 
Proof. By adding and subtracting $X_{1}+P A^{T} X_{1}^{-1} A P$ to the CARE (4), we can get

$$
\begin{gathered}
\left(P A^{T}+X_{1}\right) X_{1}^{-1}\left(P A^{T}+X_{1}\right)^{T}-P R P \\
+Q-X_{1}-P A^{T} X_{1}^{-1} A P=0
\end{gathered}
$$

which is equivalent to

$$
\begin{aligned}
0< & P\left(R+A^{T} X_{1}^{-1} A\right) P \\
& =Q-X_{1}+\left(P A^{T}+X_{1}\right) X_{1}^{-1}\left(P A^{T}+X_{1}\right)^{T} .
\end{aligned}
$$

Introducing Lemmas 1, 2, 4, 5, and 8, respectively, to (39) gives

$$
\begin{aligned}
0 & <P\left(R+A^{T} X_{1}^{-1} A\right) P \\
& =Q-X_{1}+\left(P A^{T}+X_{1}\right) X_{1}^{-1}\left(P A^{T}+X_{1}\right)^{T} \\
& \leq Q-X_{1}+\lambda_{1}\left(X_{1}^{-1}\right)\left[\left(P A^{T}+X_{1}\right)\left(P A^{T}+X_{1}\right)^{T}\right] \\
& \leq Q-X_{1}+\lambda_{1}\left(X_{1}^{-1}\right)\left[s_{1}^{2}\left(P A^{T}+X_{1}\right)\right] I \\
& \leq Q-X_{1}+\lambda_{1}\left(X_{1}^{-1}\right)\left[s_{1}\left(P A^{T}\right)+\lambda_{1}\left(X_{1}\right)\right]^{2} I \\
& \leq Q-X_{1}+\lambda_{1}\left(X_{1}^{-1}\right)\left[\lambda_{1}(P) s_{1}(A)+\lambda_{1}\left(X_{1}\right)\right]^{2} I \\
& \leq Q-X_{1}+\lambda_{1}\left(X_{1}^{-1}\right)\left[\eta s_{1}(A)+\lambda_{1}\left(X_{1}\right)\right]^{2} I .
\end{aligned}
$$

By the definition (37) of $M_{1}$ and pre- and postmultiplying $M_{1}$ to $(40)$ yields

$$
\begin{aligned}
& \left(M_{1}^{1 / 2} P M_{1}^{1 / 2}\right)^{2}=M_{1}^{1 / 2} P M_{1} P M_{1}^{1 / 2} \\
& \quad \leq M_{1}^{1 / 2}\left[Q-X_{1}+\lambda_{1}\left(X_{1}^{-1}\right)\left[\eta s_{1}(A)+\lambda_{1}\left(X_{1}\right)\right]^{2} I\right] M_{1}^{1 / 2} .
\end{aligned}
$$

Solving this inequality for $P$ shows the upper bound (36).

This builds the proof.

Remark 14. Note that for the upper bound (36), the matrices $R$ and $Q$ don not have to be nonsingular. This means that the upper bound proposed by Theorem 13 can always be computed without any condition for positive definite matrix $X_{1}$ which arbitrarily is selected.

From Theorem 13, we have the following corollaries.

Corollary 15. The positive semidefinite solution P to the CARE (4) has

$$
\begin{aligned}
P \leq\left\{\frac{1}{\lambda_{n}\left(M_{1}\right)}[Q\right. & -X_{1} \\
& \left.\left.+\lambda_{1}\left(X_{1}^{-1}\right)\left[\eta s_{1}(A)+\lambda_{1}\left(X_{1}\right)\right]^{2} I\right]\right\}^{1 / 2} \\
\equiv P_{u 2}^{1}, &
\end{aligned}
$$

where $\eta$ and $M_{1}$ for the positive definite matrix $X_{1}$ are defined by (14) and (37), respectively.
Proof. Applying Lemma 1 to the right side of (41) and solving it with regard to $P$ give the upper bound $P_{u 2}^{1}$.

Corollary 16. The solution $P$ to the CARE (4) satisfies the following upper eigenvalue bounds:

$$
\begin{aligned}
\lambda_{i}(P) & \leq \min \lambda_{i}\left(P_{u 2}\left(\eta, X_{1}, A, \mathrm{Q}, R\right)\right)=P_{u 2}^{*} \\
& \leq \lambda_{i}\left(P_{u 2}\left(\eta, X_{1}, A, \mathrm{Q}, R\right)\right), \\
\operatorname{tr}(P) & \leq \sum_{i=1}^{n} P_{u 2}^{*} \leq \min \operatorname{tr}\left(\mathrm{P}_{\mathrm{u} 2}\left(\eta, \mathrm{X}_{1}, \mathrm{~A}, \mathrm{Q}, \mathrm{R}\right)\right) \\
& \leq \operatorname{tr}\left(P_{u 2}\left(\eta, X_{1}, A, \mathrm{Q}, R\right)\right), \\
\operatorname{det}(P) & \leq \prod_{i=1}^{n} P_{u 2}^{*} \leq \min \operatorname{det}\left(\mathrm{P}_{\mathrm{u} 2}\left(\eta, \mathrm{X}_{1}, \mathrm{~A}, \mathrm{Q}, \mathrm{R}\right)\right) \\
& \leq \operatorname{det}\left(P_{u 2}\left(\eta, X_{1}, A, \mathrm{Q}, R\right)\right),
\end{aligned}
$$

where $\eta$ is defined by (14) and the positive matrix $X_{1}$ is selected so as to satisfy the definition (37), respectively.

Theorem 17. Let $P$ be the positive semidefinite solution of the CARE (4). Then $P$ has the upper bound

$$
\begin{gathered}
P \leq M_{2}^{-1 / 2}\left\{M _ { 2 } ^ { 1 / 2 } \left[Q-X_{2}+\lambda_{1}\left(X_{2}^{-1}\right) X_{2}^{2}\right.\right. \\
\left.\left.+2 \kappa_{2}\left(X_{2}\right) s_{1}(A) \varphi_{2} I\right] M_{2}^{1 / 2}\right\}^{1 / 2} M_{2}^{-1 / 2} \\
\equiv P_{u 3}\left(\varphi_{2}, X_{2}, A, Q, R\right),
\end{gathered}
$$

where the positive definite matrix $X_{2}$ is chosen so that

$$
M_{2} \equiv R-A^{T}\left[\lambda_{1}\left(X_{2}^{-1}\right) I-X_{2}^{-1}\right] A>0,
$$

and $\varphi_{2}$ is defined by

$$
\begin{gathered}
\varphi_{2} \equiv\left\{\frac{1}{\lambda_{n}\left(M_{2}\right)} \lambda_{1}\left[Q-X_{2}+\lambda_{1}\left(X_{2}^{-1}\right) X_{2}^{2}\right]\right. \\
\left.+\frac{\kappa_{2}^{2}\left(X_{2}\right) s_{1}^{2}(A)}{\lambda_{n}^{2}\left(M_{2}\right)}\right\}^{1 / 2}+\frac{\kappa_{2}\left(X_{2}\right) s_{1}(A)}{\lambda_{n}\left(M_{2}\right)} .
\end{gathered}
$$

Proof. By the use of the equality (39), from Lemmas 1 and 2, we can write

$$
\begin{aligned}
& P\left(R+A^{T} X_{2}^{-1} A\right) P \\
& \quad=Q-X_{2}+\left(P A^{T}+X_{2}\right) X_{2}^{-1}\left(P A^{T}+X_{2}\right)^{T} \\
& \quad \leq Q-X_{2}+\lambda_{1}\left(X_{2}^{-1}\right)\left[P A^{T} A P+P A^{T} X_{2}+X_{2} A P+X_{2}^{2}\right]
\end{aligned}
$$

Having applied Lemmas 1, 6, and 5, respectively, to the part of $P A^{T} X_{2}+X_{2} A P$ in (47), since the following inequalities hold:

$$
\begin{aligned}
& P A^{T} X_{2}+X_{2} A P \leq \lambda_{1}\left(P A^{T} X_{2}+X_{2} A P\right) I \\
& \quad \leq\left|\lambda_{1}\left(P A^{T} X_{2}+X_{2} A P\right)\right| I \leq 2 s_{1}\left(P A^{T} X_{2}\right) \\
& \quad \leq 2 \lambda_{1}(P) s_{1}(A) \lambda_{1}\left(X_{2}\right) I
\end{aligned}
$$


via the definition of $M_{2}$ from (47), we arrive at

$$
\begin{aligned}
P[R & \left.-A^{T}\left(\lambda_{1}\left(X_{2}^{-1}\right) I-X_{2}^{-1}\right) A\right] P \\
= & P M_{2} P \leq Q-X_{2}+\lambda_{1}\left(X_{2}^{-1}\right) X_{2}^{2} \\
& +2 \kappa_{2}\left(X_{2}\right) s_{1}(A) \lambda_{1}(P) I .
\end{aligned}
$$

Applying Lemmas 1 and 3 to (49), we have

$$
\begin{aligned}
P^{2} \leq & \frac{1}{\lambda_{n}\left(M_{2}\right)}\left[Q-X_{2}+\lambda_{1}\left(X_{2}^{-1}\right) X_{2}^{2}\right] \\
& +2 \frac{\kappa_{2}\left(X_{2}\right) s_{1}(A)}{\lambda_{n}\left(M_{2}\right)} \lambda_{1}(P) I \\
\leq & \frac{1}{\lambda_{n}\left(M_{2}\right)} \lambda_{1}\left[Q-X_{2}+\lambda_{1}\left(X_{2}^{-1}\right) X_{2}^{2}\right] I \\
& +2 \frac{\kappa_{2}\left(X_{2}\right) s_{1}(A)}{\lambda_{n}\left(M_{2}\right)} \lambda_{1}(P) I .
\end{aligned}
$$

Then,

$$
\begin{aligned}
\lambda_{1}\left(P^{2}\right) \leq & \frac{1}{\lambda_{n}\left(M_{2}\right)} \lambda_{1}\left[Q-X_{2}+\lambda_{1}\left(X_{2}^{-1}\right) X_{2}^{2}\right] \\
& +2 \frac{\kappa_{2}\left(X_{2}\right) s_{1}(A)}{\lambda_{n}\left(M_{2}\right)} \lambda_{1}(P) .
\end{aligned}
$$

Solving (51) with respect to $\lambda_{1}(P)$ gives

$$
\begin{aligned}
\lambda_{1}(P) \leq & \left\{\frac{1}{\lambda_{n}\left(M_{2}\right)} \lambda_{1}\left[Q-X_{2}+\lambda_{1}\left(X_{2}^{-1}\right) X_{2}^{2}\right]\right. \\
& \left.+\frac{\kappa_{2}^{2}\left(X_{2}\right) s_{1}^{2}(A)}{\lambda_{n}^{2}\left(M_{2}\right)}\right\}^{1 / 2}+\frac{\kappa_{2}\left(X_{2}\right) s_{1}(A)}{\lambda_{n}\left(M_{2}\right)} \equiv \varphi_{2} .
\end{aligned}
$$

Substituting $\varphi_{2}$ into (49), we get

$$
P M_{2} P \leq Q-X_{2}+\lambda_{1}\left(X_{2}^{-1}\right) X_{2}^{2}+2 \kappa_{2}\left(X_{2}\right) s_{1}(A) \varphi_{2} I .
$$

Pre- and postmultiplying $M_{2}^{1 / 2}$ to (53) leads to

$$
\begin{aligned}
& \left(M_{2}^{1 / 2} P M_{2}^{1 / 2}\right)^{2} \\
& \quad \leq M_{2}^{1 / 2}\left[Q-X_{2}+\lambda_{1}\left(X_{2}^{-1}\right) X_{2}^{2}\right. \\
& \left.\quad+2 \kappa_{2}\left(X_{2}\right) s_{1}(A) \varphi_{2} I\right] M_{2}^{1 / 2} .
\end{aligned}
$$

Therefore, by the nonsingularity of $M_{2}$, the upper matrix bound (44) is directly obtained by solving (54) with respect to $P$.

The proof is finished.

According to Theorem 17, we can propose the following corollaries.
Corollary 18. The positive semidefinite solution $P$ to the CARE (4) satisfies

$$
\begin{aligned}
P \leq\left\{\frac { 1 } { \lambda _ { n } ( M _ { 2 } ) } \left[Q-X_{2}+\lambda_{1}\left(X_{2}^{-1}\right) X_{2}^{2}\right.\right. & \\
& \left.\left.+2 \kappa_{2}\left(X_{2}\right) s_{1}(A) \varphi_{2} I\right]\right\}^{1 / 2} \equiv P_{u 3}^{1},
\end{aligned}
$$

where the positive definite matrices $X_{2}$, and $M_{2}$ and the positive constant $\varphi_{2}$ are defined by (45) and (46), respectively.

Proof. Substituting $\varphi_{2}$ into (50), having solved (50) regard to $P$, we obtain the upper bound (55).

Corollary 19. The positive semidefinite solution $P$ to the CARE (4) has the following eigenvalue upper bounds:

$$
\begin{aligned}
& \lambda_{i}(P) \leq \min \lambda_{i}\left(P_{u 3}\left(\varphi_{2}, X_{2}, A, Q, R\right)\right)=P_{u 3}^{*} \\
& \leq \lambda_{i}\left(P_{u 3}\left(\varphi_{2}, X_{2}, A, Q, R\right)\right) \\
& \operatorname{tr}(P) \leq \sum_{i=1}^{n} P_{u 3}^{*} \leq \min \operatorname{tr}\left(P_{u 3}\left(\varphi_{2}, X_{2}, A, Q, R\right)\right) \\
& \leq \operatorname{tr}\left(P_{u 3}\left(\varphi_{2}, X_{2}, A, Q, R\right)\right) \\
& \operatorname{det}(P) \leq \prod_{i=1}^{n} P_{u 3}^{*} \leq \min \operatorname{det}\left(P_{u 3}\left(\varphi_{2}, X_{2}, A, Q, R\right)\right) \\
& \leq \operatorname{det}\left(P_{u 3}\left(\varphi_{2}, X_{2}, A, Q, R\right)\right),
\end{aligned}
$$

where the positive definite matrices $X_{2}$ and $M_{2}$ and the positive constant $\varphi_{2}$ are defined by (45) and (46), respectively.

As considered a diverse matrix identity, in the case that the matrix $R$ is nonsingular, we can derive the following alternative upper bounds for the solution of the CARE (4).

Theorem 20. If the positive definite matrix $P$ is a unique solution matrix of the CARE (4), then

$$
\begin{gathered}
P \leq\left\{\frac { 1 } { \lambda _ { n } ( M _ { 3 } ) } \left[Q-A^{T} X_{3} A+\lambda_{1}\left(X_{3}^{-1}\right)\right.\right. \\
\left.\left.\times\left[\eta+s_{1}\left(A^{T} X_{3}\right)\right]^{2} I\right]\right\}^{1 / 2} \\
\equiv P_{u 4}\left(\eta, X_{3}, A, Q, R\right),
\end{gathered}
$$

where $X_{3}$ is a positive constant matrix such that $M_{3} \equiv R+$ $X_{3}^{-1}>0$ and $\eta$ is defined by (14).

Proof. When the term $P X_{3}^{-1} P+A^{T} X_{3} A$ is added and subtracted from the CARE (4), we can write

$$
\begin{aligned}
& \left(P+A^{T} X_{3}\right) X_{3}^{-1}\left(P+A^{T} X_{3}\right)^{T} \\
& \quad-P R P+Q-A^{T} X_{3} A-P X_{3}^{-1} P=0
\end{aligned}
$$


which is equivalent to

$$
\begin{aligned}
0 & <P\left(R+X_{3}^{-1}\right) P \\
& =Q-A^{T} X_{3} A+\left(P+A^{T} X_{3}\right) X_{3}^{-1}\left(P+A^{T} X_{3}\right)^{T} .
\end{aligned}
$$

By the use of Lemmas 1, 2, 4, and 8 for the right side of the above equation, respectively, we obtain

$$
\begin{aligned}
0 & <P\left(R+X_{3}^{-1}\right) P \\
& \leq Q-A^{T} X_{3} A+\lambda_{1}\left(X_{3}^{-1}\right)\left(P+A^{T} X_{3}\right)\left(P+A^{T} X_{3}\right)^{T} \\
& \leq Q-A^{T} X_{3} A+\lambda_{1}\left(X_{3}^{-1}\right) s_{1}^{2}\left(P+A^{T} X_{3}\right) I \\
& \leq Q-A^{T} X_{3} A+\lambda_{1}\left(X_{3}^{-1}\right)\left[\lambda_{1}(P)+s_{1}\left(A^{T} X_{3}\right)\right]^{2} I \\
& \leq Q-A^{T} X_{3} A+\lambda_{1}\left(X_{3}^{-1}\right)\left[\eta+s_{1}\left(A^{T} X_{3}\right)\right]^{2} I
\end{aligned}
$$

and by the application of Lemma 1 to the term $P\left(R+X_{3}^{-1}\right) P$ of (60), we can write

$$
\begin{aligned}
P^{2} \leq & \frac{1}{\lambda_{n}\left(R+X_{3}^{-1}\right)} \\
& \times\left[Q-A^{T} X_{3} A+\lambda_{1}\left(X_{3}^{-1}\right)\left[\eta+s_{1}\left(A^{T} X_{3}\right)\right]^{2} I\right] .
\end{aligned}
$$

Therefore, if the above inequality is solved with respect to $P$, we arrive at the upper bound $P_{u 4}$.

Thus, the proof is established.

Theorem 21. Let $P$ be the positive semidefinite solution of the CARE (4). Then

$$
\begin{gathered}
P \leq\left\{\frac { 1 } { \lambda _ { n } ( M _ { 4 } ) } \left[Q-A^{T} X_{4} A+\lambda_{1}\left(X_{4}^{-1}\right) A^{T} X_{4}^{2} A\right.\right. \\
\left.\left.+2 s_{1}(A) \kappa_{2}\left(X_{4}\right) \varphi_{3} I\right]\right\}^{1 / 2} \\
\equiv P_{u 5}\left(\varphi_{3}, X_{4}, A, Q, R\right),
\end{gathered}
$$

where the positive definite matrix $X_{4}$ is selected such that

$$
M_{4} \equiv R-\left[\lambda_{1}\left(X_{4}^{-1}\right) I-X_{4}^{-1}\right]>0
$$

and the nonnegative constant $\varphi_{3}$ is defined by

$$
\begin{gathered}
\varphi_{3} \equiv\left\{\frac{1}{\lambda_{n}\left(M_{4}\right)} \lambda_{1}\left[Q-A^{T} X_{4} A+\lambda_{1}\left(X_{4}^{-1}\right) A^{T} X_{4}^{2} A\right]\right. \\
\left.+\frac{\kappa_{2}^{2}\left(X_{4}\right) s_{1}^{2}(A)}{\lambda_{n}^{2}\left(M_{4}\right)}\right\}^{1 / 2}+\frac{\kappa_{2}\left(X_{4}\right) s_{1}(A)}{\lambda_{n}\left(M_{4}\right)} .
\end{gathered}
$$

Proof. Consider (58). From Lemma 1, we can easily write

$$
\begin{aligned}
0 & <P\left(R+X_{4}^{-1}\right) P \\
& \leq Q-A^{T} X_{4} A+\lambda_{1}\left(X_{4}^{-1}\right)\left(P+A^{T} X_{4}\right)\left(P+A^{T} X_{4}\right)^{T}
\end{aligned}
$$

and then via the inequality obtained by using Lemmas 1, 6, and 5 , respectively,

$$
P X_{4} A+A^{T} X_{4} P \leq 2 \lambda_{1}(P) s_{1}(A) \lambda_{1}\left(X_{4}\right)
$$

and the definition (63) of $M_{4}$, from (65), we have

$$
\begin{aligned}
0< & P\left[R-\left(\lambda_{1}\left(X_{4}^{-1}\right) I-X_{4}^{-1}\right)\right] P=P M_{4} P \\
\leq & Q-A^{T} X_{4} A+\lambda_{1}\left(X_{4}^{-1}\right) A^{T} X_{4}^{2} A \\
& +2 s_{1}(A) \kappa_{2}\left(X_{4}\right) \lambda_{1}(P) I .
\end{aligned}
$$

By the use of Lemmas 1 and 2, it is obtained that

$$
\begin{aligned}
P^{2} \leq & \frac{1}{\lambda_{n}\left(M_{4}\right)} \\
& \times\left[Q-A^{T} X_{4} A+\lambda_{1}\left(X_{4}^{-1}\right) A^{T} X_{4}^{2} A\right. \\
& \left.\quad+2 s_{1}(A) \kappa_{2}\left(X_{4}\right) \lambda_{1}(P) I\right]
\end{aligned}
$$

and thus applying Lemma 3 to (68) yields

$$
\begin{aligned}
\lambda_{1}\left(P^{2}\right) \leq & \frac{1}{\lambda_{n}\left(M_{4}\right)} \\
& \times\left\{\lambda_{1}\left[Q-A^{T} X_{4} A+\lambda_{1}\left(X_{4}^{-1}\right) A^{T} X_{4}^{2} A\right]\right. \\
& \left.+2 s_{1}(A) \kappa_{2}\left(X_{4}\right) \lambda_{1}(P)\right\} .
\end{aligned}
$$

As solving (69) according to $\lambda_{1}(P)$, one can reach the nonnegative constant $\varphi_{3}$ is defined by (64). If it is substituted $\varphi_{3}$ into (68), then

$$
\begin{gathered}
P^{2} \leq \frac{1}{\lambda_{n}\left(M_{4}\right)}\left[Q-A^{T} X_{4} A+\lambda_{1}\left(X_{4}^{-1}\right) A^{T} X_{4}^{2} A\right. \\
\left.+2 s_{1}(A) \kappa_{2}\left(X_{4}\right) \varphi_{3} I\right] .
\end{gathered}
$$

Thus, solving the inequality (70) derives the upper bound (62) for the solution $P$ of the CARE (4).

This concludes the proof of the theorem.

Corollary 22. The solution $P$ to the CARE (4) has the following eigenvalue bounds for $j=4,5$ :

$$
\begin{gathered}
\lambda_{i}(P) \leq \min \lambda_{i}\left(P_{u j}\right)=P_{u j}^{*} \leq \lambda_{i}\left(P_{u j}\right), \\
\operatorname{tr}(P) \leq \sum_{i=1}^{n} P_{u j}^{*} \leq \min \operatorname{tr}\left(P_{u j}\right) \leq \operatorname{tr}\left(P_{u j}\right), \\
\operatorname{det}(P) \leq \prod_{i=1}^{n} P_{u j}^{*} \leq \min \operatorname{det}\left(P_{u j}\right) \leq \operatorname{det}\left(P_{u j}\right) .
\end{gathered}
$$

Remark 23. Chen and Lee in [16] indicated in it is hard or impossible to determine the best matrix bound among the parallel results. Since we find that it is difficult to compare the tightness of our results to the parallel result in [18], we will only make the comparisons on an example. 


\section{Numerical Example}

In this section, we will give a numerical example to demonstrate the effectiveness of the proposed results of this paper.

Example 1. Consider the CARE (4) with

$$
A=\left(\begin{array}{cc}
-1 & 0 \\
1 & 0
\end{array}\right), \quad Q=\left(\begin{array}{ll}
1 & 0 \\
0 & 8
\end{array}\right), \quad R=\left(\begin{array}{cc}
9 & 4 \\
4 & 16
\end{array}\right) .
$$

Choose $\varepsilon=0.002$, then using (16) shows the following upper matrix bound:

$$
\begin{gathered}
P \leq\left(\begin{array}{cc}
0.8232 & -0.0001 \\
-0.0001 & 1.2853
\end{array}\right) \equiv P_{u 1}, \\
\operatorname{tr}\left(P_{u 1}\right)=2.1085, \quad \operatorname{det}\left(P_{u 1}\right)=1.0580 .
\end{gathered}
$$

The upper bound (55) gives

$$
\begin{gathered}
P \leq\left(\begin{array}{ll}
0.8173 & 0.0068 \\
0.0068 & 1.2815
\end{array}\right) \equiv P_{u 3}^{1}, \\
\operatorname{tr}\left(P_{u 3}^{1}\right)=2.0988, \\
\operatorname{det}\left(P_{u 3}^{1}\right)=1.0473,
\end{gathered}
$$

with $X_{2}=\left(\begin{array}{cc}10 & 0.1 \\ 0.1 & 10\end{array}\right)$, and the upper bound (62) gives

$$
\begin{gathered}
P \leq\left(\begin{array}{cc}
0.8070 & 0 \\
0 & 1.2750
\end{array}\right) \equiv P_{u 5}, \\
\operatorname{tr}\left(P_{u 5}\right)=2.082 \\
\operatorname{det}\left(P_{u 5}\right)=1.0289,
\end{gathered}
$$

with $X_{4}=\left(\begin{array}{cc}10 & 0.1 \\ 0.1 & 10\end{array}\right)$.

Using Theorem 3.1 of [19], we obtain the following upper matrix bound:

$$
\begin{gathered}
P \leq\left(\begin{array}{ll}
1.2328 & 0.0002 \\
0.0002 & 1.2884
\end{array}\right) \equiv P_{u[19]}, \\
\operatorname{tr}\left(P_{u[19]}\right)=2.5212, \\
\operatorname{det}\left(P_{u[19]}\right)=1.5883 .
\end{gathered}
$$

The bounds (18) and (23) proposed in [18] are

$$
\begin{gathered}
P \leq\left(\begin{array}{cc}
1.7135 & 0 \\
0 & 1.0627
\end{array}\right) \equiv P_{u 1[18]}, \\
\operatorname{tr}\left(P_{u 1[18]}\right)=2.7762, \\
\operatorname{det}\left(P_{u 1[18]}\right)=1.8209, \\
P \leq\left(\begin{array}{ll}
1.2527 & 0.0050 \\
0.0050 & 1.6025
\end{array}\right) \equiv P_{u 2[18]}, \\
\operatorname{tr}\left(P_{u 2[18]}\right)=2.8552, \\
\operatorname{det}\left(P_{u 2[18]}\right)=2.0074,
\end{gathered}
$$

for $\bar{R}_{1}=X_{4}$.
By a simple computation, we have

$$
\begin{gathered}
P_{u 5} \leq P_{u 3}^{1} \leq P_{u[19]} \leq P_{u 2[18]}, \\
P_{u 5} \leq P_{u 1} \leq P_{[19]}, \\
\operatorname{tr}\left(P_{u 5}\right) \leq \operatorname{tr}\left(P_{u 3}^{1}\right) \leq \operatorname{tr}\left(P_{u 1}\right) \leq \operatorname{tr}\left(P_{u[19]}\right), \\
\leq \operatorname{tr}\left(P_{u 1[18]}\right) \leq \operatorname{tr}\left(P_{u 2[18]}\right), \\
\operatorname{det}\left(P_{u 5}\right) \leq \operatorname{det}\left(P_{u 3}^{1}\right) \leq \operatorname{det}\left(P_{u 1}\right) \leq \operatorname{det}\left(P_{u[19]}\right), \\
\leq \operatorname{det}\left(P_{u 1[18]}\right) \leq \operatorname{det}\left(P_{u 2[18]}\right)
\end{gathered}
$$

which means that our upper bounds give more precise solution estimates than the results given by Theorem 3.1 in [19] and Theorems 2 and 3 in [18] for this case.

\section{Conclusion}

In this paper, new upper matrix bounds for the solution of the CARE are improved by using some linear algebraic techniques and matrix inequalities. A numerical example is given to show that the solution upper bounds presented in this paper are sharper than some results in the literature.

\section{Acknowledgments}

The authors would like to thank the editor and the reviewers for the very helpful comments and suggestions to improve the presentation of this study. This study has been supported by the Coordinatorship of Selçuk University's Scientific Research Projects (BAP) and The Scientific and Technical Research Council of Turkey (TUBITAK).

\section{References}

[1] M. Basin, J. Rodriguez-Gonzalez, and L. Fridman, "Optimal and robust control for linear state-delay systems," Journal of the Franklin Institute, vol. 344, no. 6, pp. 830-845, 2007.

[2] R. Davies, P. Shi, and R. Wiltshire, "New upper solution bounds for perturbed continuous algebraic Riccati equations applied to automatic control," Chaos, Solitons and Fractals, vol. 32, no. 2, pp. 487-495, 2007.

[3] H. Kwakernaak and R. Sivan, Linear Optimal Control Systems, Wiley-Interscience, New York, NY, USA, 1972.

[4] S. S. Wang, B. S. Chen, and T. P. Lin, "Robust stability of uncertain time-delay systems," International Journal of Control, vol. 46, no. 3, pp. 963-976, 1987.

[5] X. Su, P. Shi, L. Wu, and Y. D. Song, "A novel approach to filter design for T-Sfuzzy discrete time systems with time-varying delay," IEEE Transactions on Fuzzy Systems, vol. 20, no. 6, pp. 1114-1129, 2012.

[6] S. Barnett and C. Storey, Matrix Methods in Stability Theory, Barnes and Noble Inc., New York, NY, USA, 1970.

[7] T. M. Huang and W. W. Lin, "Structured doubling algorithms for weakly stabilizing Hermitian solutions of algebraic Riccati equations," Linear Algebra and Its Applications, vol. 430, no. 5-6, pp. 1452-1478, 2009. 
[8] L. Wu, X. Su, P. Shi, and J. Qiu, "A new approach to stability analysis and stabilization of discrete-time T-S fuzzy timevarying delay systems," IEEE Transactions on Systems, Man, and Cybernetics B, vol. 41, no. 1, pp. 273-286, 2011.

[9] C. H. Lee, "Simple stabilizability criteria and memoryless state feedback control design for time-delay systems with timevarying perturbations," IEEE Transactions on Circuits and Systems I, vol. 45, no. 11, pp. 1211-1215, 1998.

[10] T. Mori and I. A. Derese, "A brief summary of the bounds on the solution of the algebraic matrix equations in control theory," International Journal of Control, vol. 39, no. 2, pp. 247-256, 1984.

[11] K. Ogata, Modern Control Engineering, Prentice-Hall, Upper Saddle River, NJ, USA, 3rd edition, 1997.

[12] W. Zhang, H. Su, H. Wang, and Z. Han, "Full-order and reduced-order observers for one-sided Lipschitz nonlinear systems using Riccati equations," Communications in Nonlinear Science and Numerical Simulation, vol. 17, no. 12, pp. 4968-4977, 2012.

[13] M. L. Ni, "Existence condition on solutions to the algebraic Riccati equation," Acta Automatica Sinica, vol. 34, no. 1, pp. 85-87, 2008.

[14] W. H. Kwon, Y. S. Moon, and S. C. Ahn, "Bounds in algebraic Riccati and Lyapunov equations: a survey and some new results," International Journal of Control, vol. 64, no. 3, pp. 377389, 1996.

[15] C. H. Lee, "New results for the bounds of the solution for the continuous Riccati and Lyapunov equations," IEEE Transactions on Automatic Control, vol. 42, no. 1, pp. 118-123, 1997.

[16] C. Y. Chen and C. H. Lee, "Explicit matrix bounds of the solution for the continuous Riccati equation," ICIC Express Letters, vol. 3, no. 2, pp. 147-152, 2009.

[17] S. W. Kim and P. G. Park, "Upper bounds of the continuous ARE solution," IEICE Transactions on Fundamentals of Electronics Communications and Computer Sciences, vol. 83, pp. 380-385, 2000.

[18] C. H. Lee, "Solution bounds of the continuous Riccati matrix equation," IEEE Transactions on Automatic Control, vol. 48, no. 8, pp. 1409-1413, 2003.

[19] J. Zhang and J. Liu, "Matrix bounds for the solution of the continuous algebraic Riccati equation," Mathematical Problems in Engineering, vol. 2010, Article ID 819064, 15 pages, 2010.

[20] W. Zhang, H. Su, and J. Wang, "Computation of upper bounds for the solution of continuous algebraic Riccati equations," Circuits, Systems and Signal Processing, vol. 32, no. 3, pp. 1477-1488, 2013.

[21] H. H. Choi and T. Y. Kuc, "Lower matrix bounds for the continuous algebraic Riccati and Lyapunov matrix equations," Automatica, vol. 38, no. 8, pp. 1147-1152, 2002.

[22] V. R. Karanam, "A note on eigenvalue bounds in algebraic Riccati equation," IEEE Transactions on Automatic Control, vol. 28, no. 1, pp. 109-111, 1983.

[23] K. Yasuda and K. Hirai, "Upper and lower bounds on the solution of the algebraic Riccati equation," IEEE Transactions on Automatic Control, vol. 24, no. 3, pp. 483-487, 1979.

[24] B. H. Kwon, M. J. Youn, and Z. Bien, "On bounds of the Riccati and Lyapunov matrix equations," IEEE Transactions on Automatic Control, vol. 30, no. 11, pp. 1134-1135, 1985.

[25] S. D. Wang, T. S. Kuo, and C. F. Hsu, "Trace bounds on the solution of the algebraic matrix Riccati and Lyapunov equation," IEEE Transactions on Automatic Control, vol. 31, no. 7, pp. 654656, 1986.
[26] R. V. Patel and M. Toda, "On norm bounds for algebraic Riccati and Lyapunov equations," IEEE Transactions on Automatic Control, vol. 23, no. 1, pp. 87-88, 1978.

[27] D. S. Bernstein, Matrix Mathematics: Theory, Facts and Formulas with Application to Linear Systems Theory, Princeton University Press, Princeton, NJ, USA, 2005.

[28] R. A. Horn and C. R. Johnson, Matrix Analysis, Cambridge University Press, Cambridge, UK, 1985.

[29] A. W. Marshall and I. Olkin, Inequalities: Theory of Majorization and Its Applications, vol. 143 of Mathematics in Science and Engineering, Academic Press, New York, NY, USA, 1979.

[30] E. Kreindler and A. Jameson, "Conditions for nonnegativeness of partitioned matrices," IEEE Transactions on Automatic Control, vol. 17, pp. 147-148, 1972. 


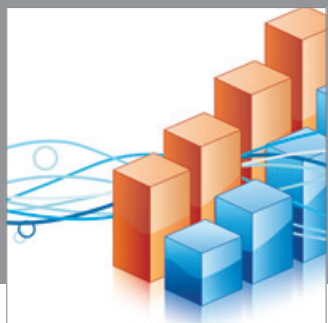

Advances in

Operations Research

mansans

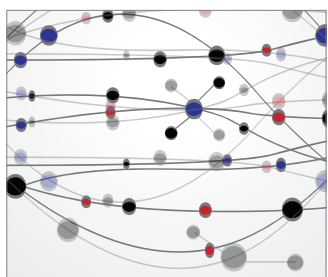

The Scientific World Journal
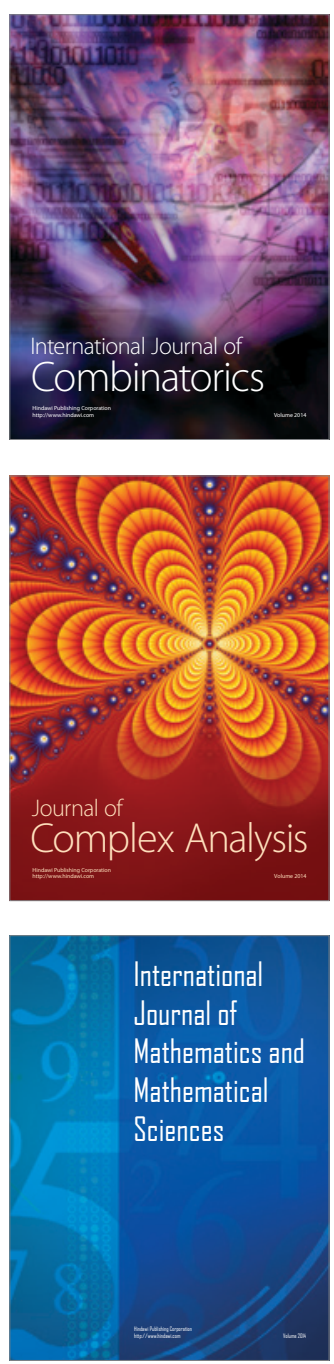
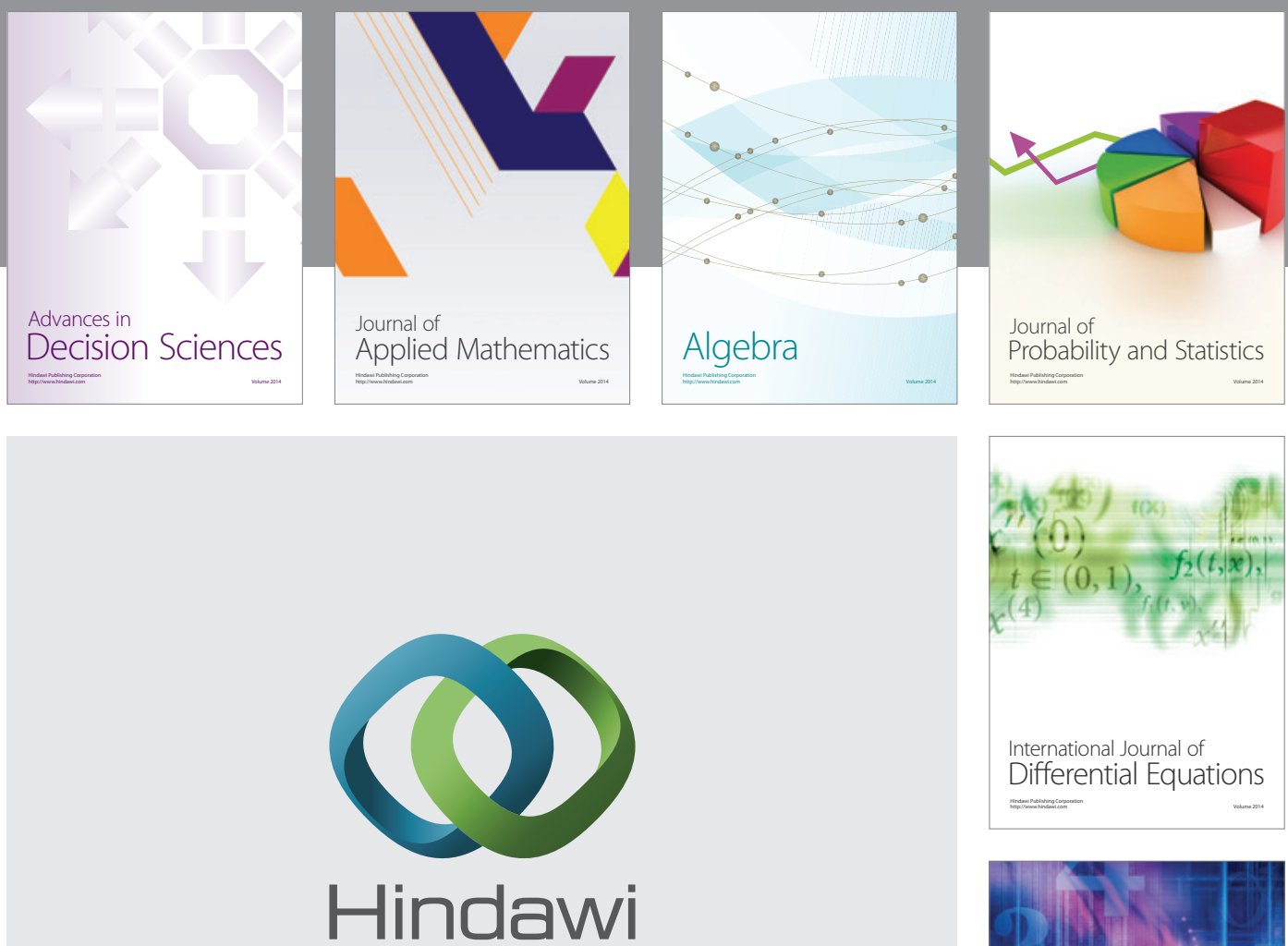

Submit your manuscripts at http://www.hindawi.com
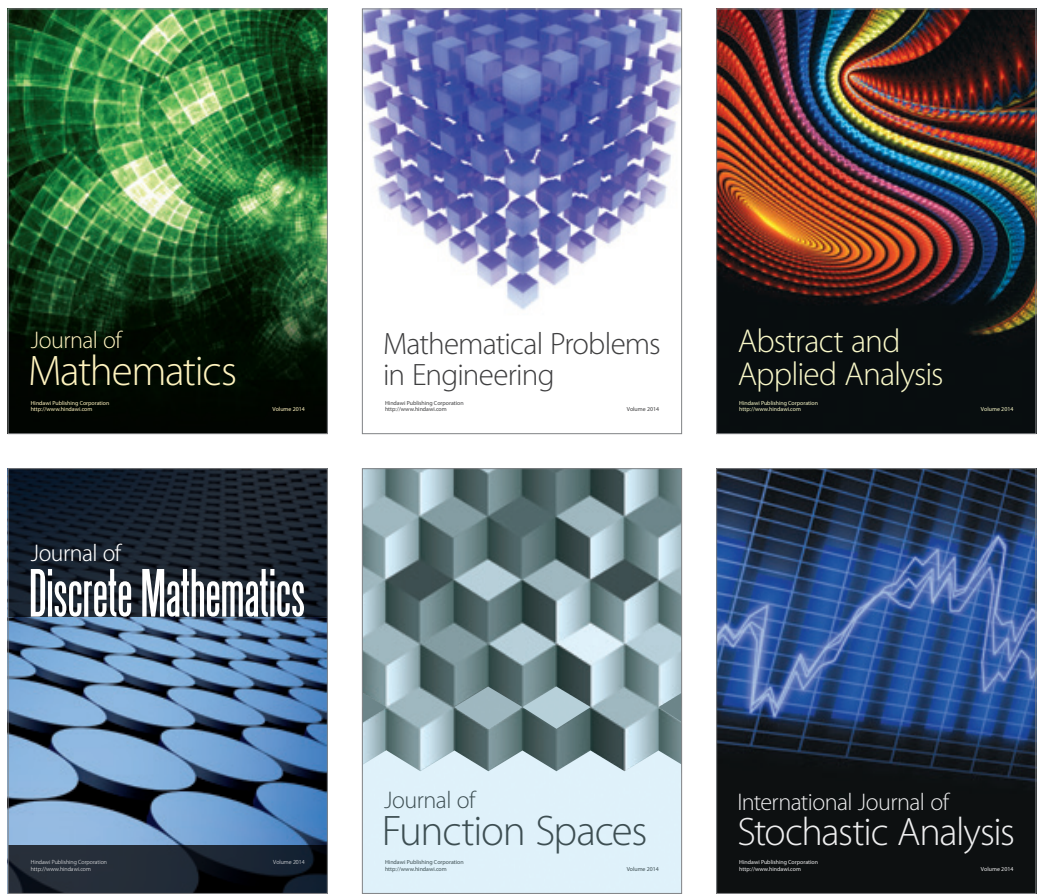

Journal of

Function Spaces

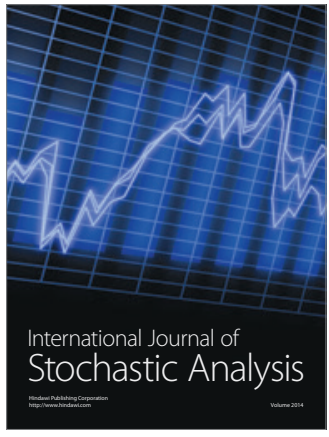

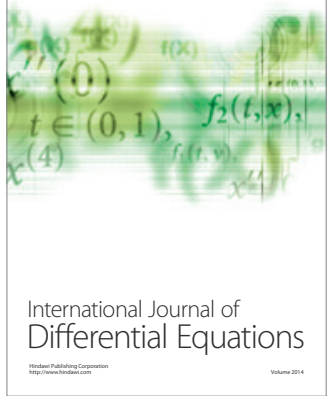
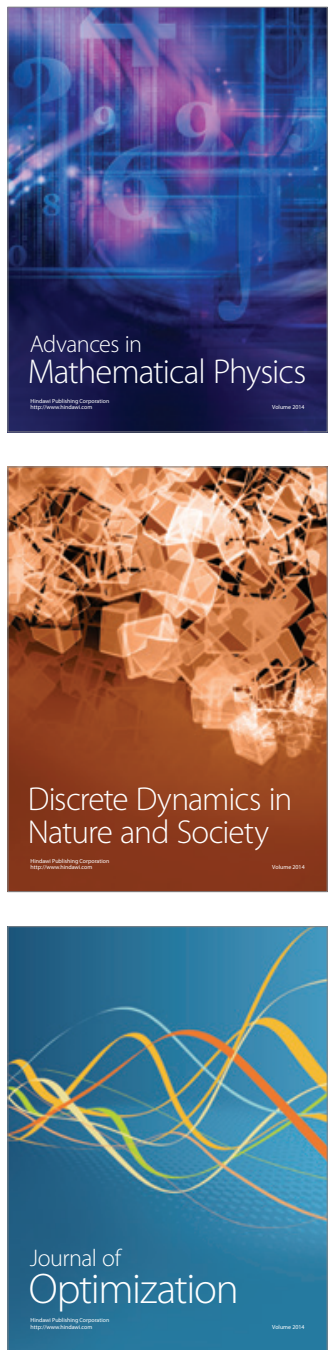